\title{
EFFECT OF TWO TYPES OF NON-MAYWELLIAN ELECTRON DISTRIBUTIONS ON TEMPERATURE SPECTROSCOPIC DIAGNOSTICS
}

C. Möller and M. Lamoureux

Laboratoire de Spectroscopie Atomique et Ionique, Université Paris-Sud, 91405, Orsay, France

Continuum emission due to bremsstrahlung and direct radiative recombination is studied in two types of fusion-plasmas with nonMaxwellian electron distributions. The calculated emissivity coefficients $J_{\omega}(K \omega)$ are shown to depart from the Maxwellian ones. The main consequence of the modification of the spectrum is to invalidate the usual electron temperature diagnostic which consits in equating

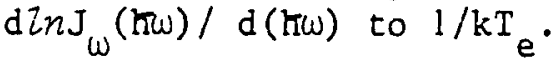

Case I corresponds to the critical density region of a laserproduced plasma (Langdon 1980, Mora and Yahi 1982). The distribution function is $c_{m} \exp (-v / v, m)^{m}$ with $m=5$ for all values of the incident electron $\mathrm{velocity} v .^{\circ}, \mathrm{m}$ Case II corresponds to very steep temperature gradients for which the spacial scale of the transport region is larger than the gradient scale itself (Albritton 1983, Luciani et al. 1984, Campbell 1984 ). On the higher temperature side of the heat front, the tail of the distribution is depleted with respect to the Maxwellian one. At a particular distance from it, this tail can be roughly described by the above analytical function but with $m=3$.

Universal emissivity curves ( Lamoureux et al. 1984 ) were given in case $I$, for completely stripped ions, for bremsstrahlung and recombination separately, provided Kramers' atomic data are suitable. Another set of curves can be similarly established in case II as well. Results can be extended to less strongly ionized plasmas by employing an efficient degree of ionization ( Kim and Pratt, 1983), $\left(\mathrm{Z}+\mathrm{Z}_{\mathrm{i}}\right) / 2$, for the captures into each ion $\mathrm{A}^{2}{ }^{+}$. Results concerning bremsstrahlung are given in Fig. 1 ; the departure of the two nonMaxwellian curves from the Maxwellian straight line reflects the nonMaxwellian character of the distribution, and the variability of their slope points out the failure of the usual diagnostic in most regions of the spectrum.

We then evaluate as an example the total emissivity coefficient $\mathrm{J}_{(}\left(\bar{n} \omega_{2}\right)$ produced at the kinetic temperature of $1 \mathrm{keV}$ and the density of $10^{21} \mathrm{e} / \mathrm{cc}$ in an Aluminium plasma consisting of $25 \% \mathrm{Al}, 50 \%$ of $\mathrm{Al}^{12+}$ and $25 \%$ of $\mathrm{Al}^{1 \mathrm{ll}}$. (See Fig. 2). In case II, non-Maxwellian effects are felt only at high photon energies. In case $I$, the nonMaxwellian character is more pronounced; if $j$ s found over the entire spectrum, and especially right above the $A 1$ is threshold, as was observed experimentally (Matthews et al. 1983). The usual temperature diagnostic is then very mileading as is illustrated by the dotted curve of Fig. 2 . 


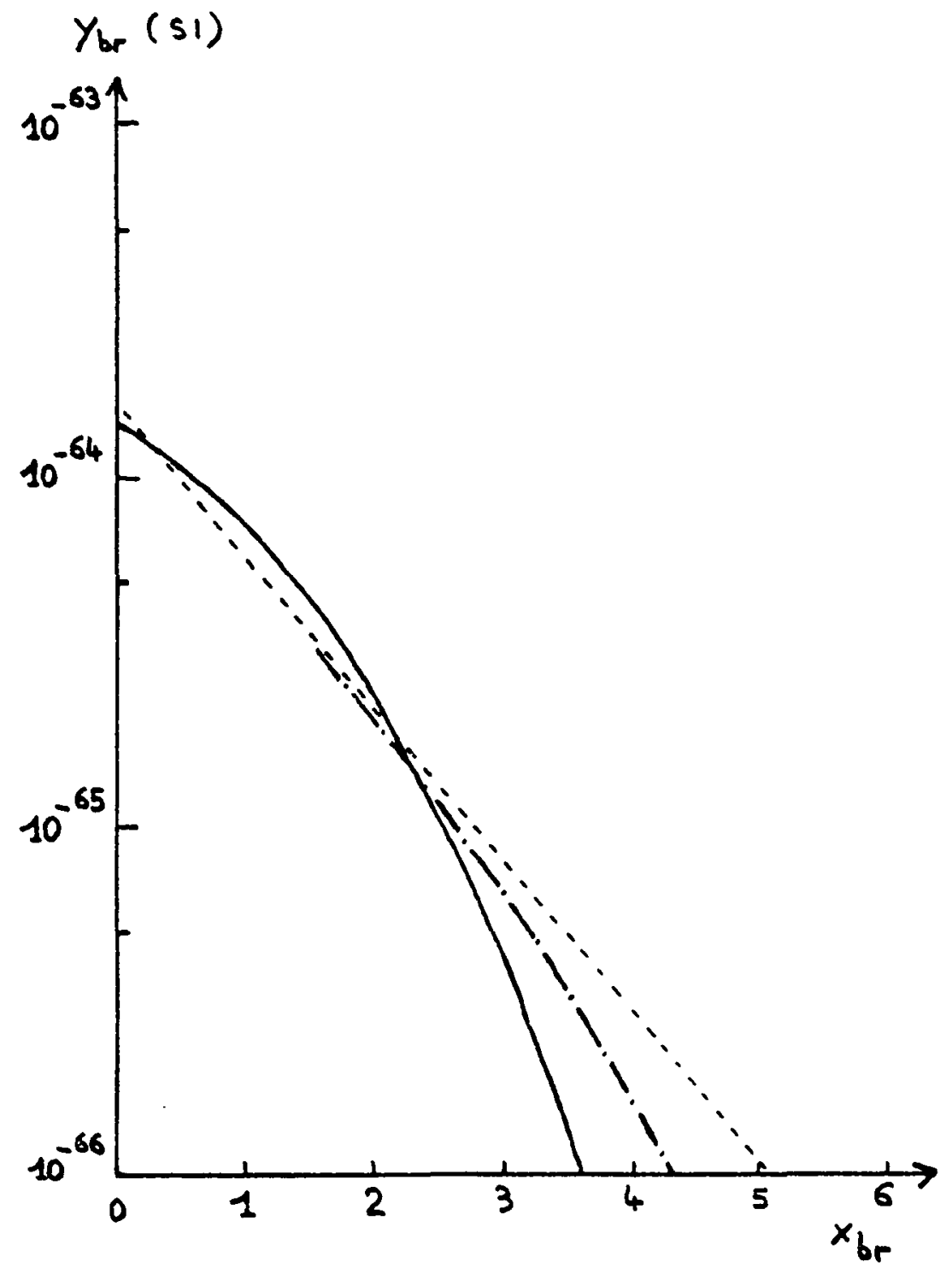

Fig. 1 : Reduced emissivity for bremsstrahlung $y_{\text {br }}$ versus photon energy $\mathrm{x}_{\mathrm{br}}=\mathrm{n} \omega / \mathrm{kT} \mathrm{T}_{\mathrm{e}} \cdot \mathrm{y}_{\mathrm{br}}$ is related to the emissivity coefficient $j_{b r}$ by $y_{b r}(\hbar \omega)=j_{b r}(\hbar \omega)(k T e)^{l / 2} /\left(N_{e} N_{i} z_{i}{ }^{2}\right)$. ( - - ) : Maxwellian distribution, ( - ) : non-Maxwellian, case I; ( - - ) : non-Maxwellian, case II. 


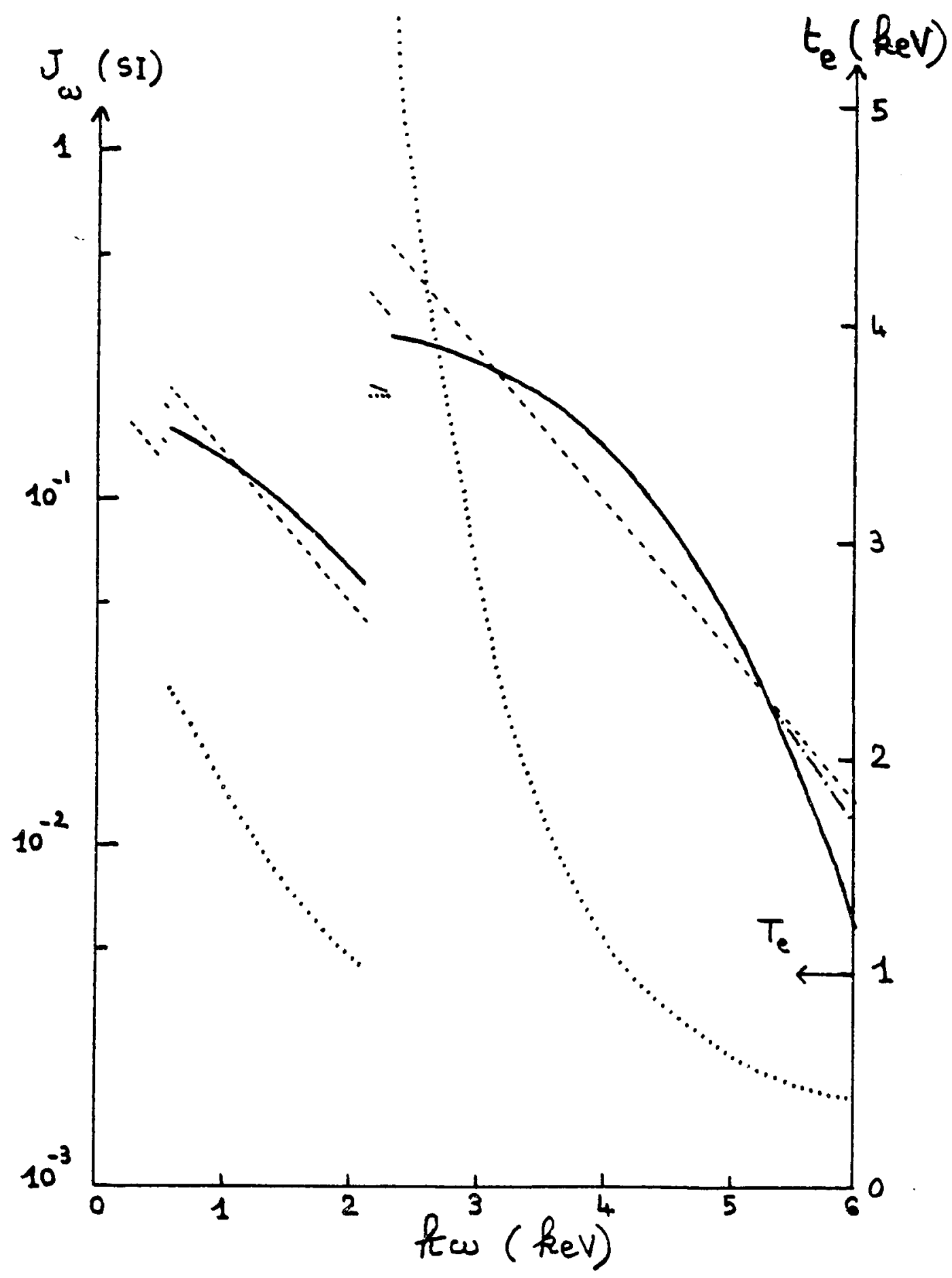

Fig. 2 : Total emissivity coefficient $J_{\omega}$ versus photon energy $\hbar \omega$ for an Al plasma, at $\mathrm{T}_{\mathrm{e}}=1 \mathrm{keV}$ and $\mathrm{d}=10^{21} \mathrm{e} / \mathrm{cc}$, consisting of $25 \% \mathrm{Al}{ }^{13+}$, $50 \% \mathrm{Al}^{12+}$ and $25 \% \mathrm{Al}^{1 \mathrm{l}+}$. ( - - ) : Maxwellian distribution, ( - ) : non-Maxwellian, case I, ( - . ) :non-Maxwellian, case II.

iisleading temperature $t$ obtained from the diagnostic whereas the kinetic temperature is $\mathrm{T}_{\mathrm{e}}^{\mathrm{e}},(\ldots)$ : non-Maxwellian distribution, case I. ( Dotted curves should be read on the right ordinate). 


\section{REFERENCES}

Albritton, J.R. 1983, Phys. Rev. Lett., 50, 2078.

Campbe11, P.M. 1984, Phys. Rev. A, 30, 365.

Young Soon Kim, and Pratt, R.H. 1983, Phys. Rev. A, 27, 2913.

Lamoureux, M., Möller, C., and Jaeglé, P. 1984, Phys. Rev. A, 30, 429.

Langdon, A.B. 1980, Phys. Rev. Lett., 44, 575.

Luciani,J., Mora, P., and Pellat, P., to be published, and private communication.

Mora, P., and Yahi, H. 1982, Phys. Rev. A, 26, 2259.

Matthews, D. L., Kauffmann, R.L., Kilkenny, J.D., and Lee, R.W. 1983, Futherford Appleton Laboratory Report No. RL83-043.

Acknowledgement : We want to thank Dr. P. Jaeglé for encouragement in this work. 